\title{
Dioscin inhibits adipogenesis through the AMPK/MAPK pathway in 3T3-L1 cells and modulates fat accumulation in obese mice
}

\author{
BARUN POUDEL ${ }^{1 *}$, SEONG-WON LIM $^{1 *}$, HYEON-HUI KI $^{1}$, \\ SARMILA NEPALI ${ }^{1}$, YOUNG-MI LEE ${ }^{2}$ and DAE-KI KIM ${ }^{1}$ \\ ${ }^{1}$ Department of Immunology and Institute of Medical Sciences, Chonbuk National University Medical School, \\ Jeonju, Jeonbuk 561-756; ${ }^{2}$ Department of Oriental Pharmacy, College of Pharmacy and Wonkwang-Oriental \\ Medicines Research Institute, Wonkwang University, Iksan, Jeonbuk 570-749, Republic of Korea
}

Received March 10, 2014; Accepted August 28, 2014

DOI: $10.3892 /$ ijmm.2014.1921

\begin{abstract}
Dioscin (DS) is a steroidal saponin present in a number of medicinal plants and has been shown to exert anticancer, antifungal and antiviral effects. The present study aimed to deternube the effects DS on the regulation of adipogenesis and to elucidate the underlying mechanisms. In vitro experiments were performed using differentiating 3T3-L1 cells treated with various concentrations $(0-4 \mu \mathrm{M})$ of DS for 6 days. A cell viability assay was performed on differentiating cells following exposure to DS. Oil Red O staining and triglyceride content assay were performed to evaluate the lipid accumulation in the cells. We also carried out the following experiments: i) flow cytometry for cell cycle analysis, ii) quantitative reverse transcription polymerase chain reaction for measuring adipogenesis-related gene expression, and iii) western blot analysis to measure the expression of adipogenesis transcription factors and AMP-activated protein kinase (AMPK), acetyl-CoA carboxylase (ACC) and mitogen-activated protein kinase (MAPK) phosphorylation. In vivo experiements were performed using mice with obesity induced by a high-fat diet (HFD) that were treated with or without DS for 7 weeks. DS suppressed lipid accumulation in the 3T3-L1 cells without affecting viability at a dose of up to $4 \mu \mathrm{M}$. It also delayed cell cycle progression $48 \mathrm{~h}$ after the initiation of adipogenesis. DS inhibited adipocyte differentiation by the downregulation of adipogenic transcription factors and attenuated the expression of adipogenesis-associated genes. In addition, it enhanced
\end{abstract}

Correspondence to: Professor Dae-Ki Kim, Department of Immunology and Institute of Medical Sciences, Chonbuk National University Medical School, 567 Baekje-daero, Deokjin-gu, Jeonju, Jeonbuk 561-756, Republic of Korea

E-mail: daekim@jbnu.ac.kr

${ }^{*}$ Contributed equally

Key words: dioscin, obesity, adipogenesis, 3T3-L1, AMP-activated protein kinase, mitogen-activated protein kinases, high-fat diet the phosphorylation of AMPK and its target molecule, ACC, during the differentiation of the cells. Moreover, the inhibition of adipogenesis by DS was mediated through the suppression of the phosphorylation of MAPKs, such as extracellular-regulated kinase 1/2 (ERK1/2) and p38, but not c-Jun-N-terminal kinase (JNK). DS significantly reduced weight gain in the mice with HFD-induced obesity; this was evident by the suppression of fat accumulation in the abdomen. The present study reveals an anti-adipogenic effect of DS in vitro and in vivo and highlights AMPK/MAPK signaling as targets for DS during adipogenesis.

\section{Introduction}

Obesity is the most common metabolic disease worldwide. It is characterized by an excessive storage of body fat, and is associated with a number of metabolic complications, including type 2 diabetes, hypertension and cardiovascular diseases (CVD) (1). Such complications could lead to higher mortality rates in obese as opposed to lean patients.

Adipogenesis contributes to excess fat deposition in adipocytes during the differentiation process from preadipocytes. The molecular and cellular mechanisms of adipogenesis have been extensively studied using the 3T3-L1 preadipocyte cell line as these cells differentiate into adipocytes upon stimulation; this process is similar to the development of obesity in humans (2-5). Adipogenesis involves concerted transcriptional and cellular events, including growth arrest, re-entry into the cell cycle for mitotic clonal expansion (MCE) and the start of transcription during differentiation (2). Numerous genes have been shown to be involved in the development of obesity. For instance, peroxisome proliferator-activated receptor $\gamma(\mathrm{PPAR} \gamma), \mathrm{CCAAT} /$ enhancer-binding protein $(\mathrm{C} / \mathrm{EBP}) \beta, \mathrm{C} / \mathrm{EBP} \alpha, \mathrm{C} / \mathrm{EBP} \delta$ and sterol regulatory element binding protein-1c (SREBP-1c) $(2,6)$. Furthermore, AMP-activated protein kinase (AMPK) has been suggested to function as a sensor of cellular energy status and, when activated, accelerates the ATP-producing catabolic pathways and simultaneously reduces the anabolic pathways that consume ATP $(6,7)$. Several studies have identified AMPK signaling as the target for the treatment of obesity and diabetes (8-10). Moreover, mitogen-activated protein kinases (MAPKs), namely, extracellular regulated kinase 1/2 (ERK 1/2), p38 and c-Jun-N-terminal kinase (JNK) are known to play a 
crucial role in several cellular processes, including adipocyte differentiation (11).

Dioscin (DS) is a steroidal saponin present in a number of medicinal plants, such as Dioscorea nipponica Makino and Dioscorea zingiberensis Wright. Traditionally, saponins from Dioscorea plants are used to treat cardiovascular diseases, rheumatoid arthritis, asthma and hyperlipidemia (12). Several studies have demonstrated that DS exerts anticancer (13-15), antifungal (16) and antiviral effects (17). A previous study reported that rats fed a high-fat diet (HFD) containing 5\% Dioscorea nipponica Makino presented with reduced weight and adipose tissue gain when compared to the control group (18). Similarly, mice fed an HFD containing the aqueous extract of the Dioscorea plant, Rhizoma Dioscoreae Tokoronis, showed lower body weight and adipose tissue in comparison to the control mice (19), suggesting that Dioscorea plants have anti-obesity effects. A recent study reported that a steroidal saponin, pseudoprotodioscin, present in the Dioscorea plant, inhibited adipogenesis in 3T3-L1 cells (12). However, the potential role of DS, an active compound in the Dioscorea plant, in adipogenesis and its underlying mechanisms of action have not yet been fully elucidated.

\section{Materials and methods}

Materials and reagents. DS (PubChem CID: 119245) (Fig. 1, adopted from http://www.ncbi.nlm.nih.gov/pccompound) was obtained from the Nanjing Zelang Medical Technology Co., Ltd., (Jiangsu, China) with $98 \%$ purity as determined by high performance liquid chromatography, and was dissolved in demethyl sulfoxide (DMSO) (Sigma, St. Louis, MO, USA) for the experiments. Dexamethasone, insulin, propidium iodide, 3-isobutyl-1-methylxanthine (IBMX), RNase A, orlistat and Oil Red O staining solution were purchased from Sigma. Dulbecco's modified Eagle's medium (DMEM) and fetal calf serum (FCS) were from HyClone (Logan, UT, USA). Zoletil 50 was obtained from Virbac Laboratories (06516 Carros, France). TRIzol reagent and the SuperScript II kit were obtained from Invitrogen (Carlsbad, CA, USA). The Cell Counting Kit-8 (CCK-8) was purchased from Dojindo Molecular Technologies (Rockville, MD, USA). Anti-AMPK, anti-p-AMPK, anti-ACC, anti-phosphorylated (p)-ACC, anti-PPAR $\gamma$, anti-C/EBP $\alpha$, anti-C/EBP $\beta$, anti-C/EBPס, anti-ERK, anti-p-ERK, anti-p38, anti-p-p38, anti-JNK and anti-p-JNK antibodies were purchased from Cell Signaling Biotechnology (Beverly, MA, USA). The protein assay kit (RIPA buffer), rabbit and mouse secondary antibodies, and anti- $\beta$-actin antibodies were obtained from Santa Cruz Biotechnology (Santa Cruz, CA, USA).

Animals and diet. C57BL/6J male mice (5 weeks old) were purchased from Samtako Inc. (Seoul, Korea). The animals were maintained in a room under the following conditions: 12-h light/dark cycles, a temperature pf $22 \pm 2^{\circ} \mathrm{C}$ and a relative humidity of $50 \pm 5 \%$ during the whole experimenta period. Mice were fed a normal diet for 2 weeks for adaptation. Subsequently, they were randomly divided into 5 groups $(n=8)$. Two groups were fed either a normal diet (normal control, NC) or an HFD (kcal\%, protein 20, carbohydrate 35, fat 45; D12451 Research Diets Inc., New Brunswick, NJ, USA). The other groups were fed an HFD and were intragastrically adminstered with DS

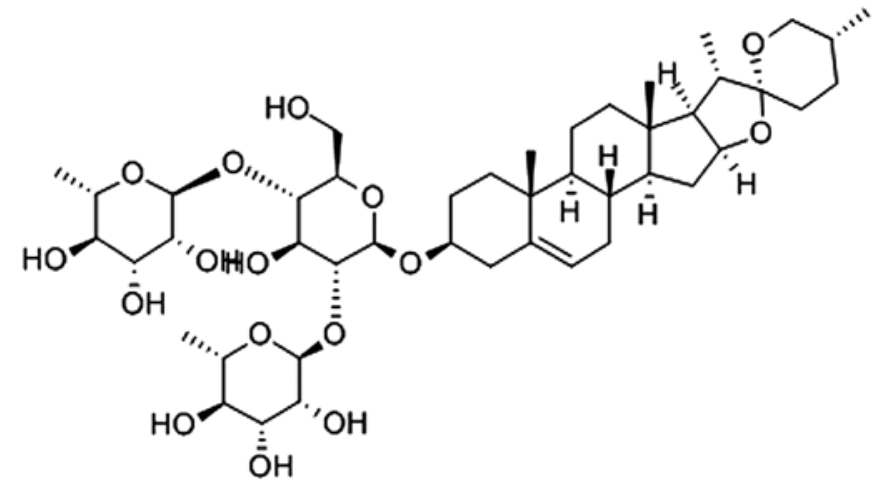

Figure 1. Chemical structure of dioscin.

or orlistat at the indicated concentrations dissolved in $0.5 \%$ carboxymethyl cellulose (CMC) in distilled water. This solution was freshly prepared each day prior to administration. The mice in the control group were administered $0.5 \% \mathrm{CMC}$. The mice were treated as described above for 7 weeks. Body weight was measured each week. All animal experiments were approved by the Institutional Animal Care and Use Committee at Chonbuk National University, Jeonju, Korea.

Determination of body fat composition by microtomoraphy or computed tomography (micro-CT) and abdominal fat isolation. The mice were starved for $6 \mathrm{~h}$ and in vivo microCT images of the anesthetized mice [Zoletil $50,2 \mathrm{mg} / \mathrm{kg}$, intraperitoneally (i.p.)] were acquired using a Skyscan-1076 micro-CT scanner (Skyscan, Aartselaar, Belgium). CT was performed using the following parameters: pixel size, $18 \mu \mathrm{m}$; source voltage, $48 \mathrm{kVp}$; and source current, $200 \mu \mathrm{A}$. The X-ray detector comprised a 12-bit water-cooled charge-coupled device high-resolution $(4,000 \times 2,300$-pixel) camera and an $\mathrm{X}$-ray scintillator. The images were acquired in increments of 0.6 degrees. The exposure time for each view was $0.46 \mathrm{sec}$; a $0.5-\mathrm{mm}$ aluminum energy filter was used.

Following micro-CT scanning, the mice were sacrificed by exposure to diethyl-ether, abdominal fat was isolated and images were acquired using an Olympus SP-500UZ camera (Olympus, Center Valley, PA, USA).

Cell culture and differentiation. 3T3-L1 preadipocytes were obtained from the American Type Culture Collection and maintained in DMEM containing $10 \%$ FCS in a humidified atmosphere of $5 \% \mathrm{CO}_{2}$ at $37^{\circ} \mathrm{C}$. The differentiation of the preadipocytes was induced 2 days post-confluence (day 0 ) by the addition $0.5 \mathrm{mM}$ IBMX, $1 \mu \mathrm{M}$ dexamethasone and $10 \mu \mathrm{g} / \mathrm{ml}$ insulin [multiple daily insulin (MDI)] for 2 days. Subsequently, the culture medium was changed to DMEM/10\% FCS containing insulin. After 2 days, the medium was replaced with DMEM $/ 10 \%$ FCS and the cells were incubated for a further 2 days. DS was added on day 0 during differentiation until the cells were harvested for the experiments described below.

Cell viability. The cells were treated with MDI and various concentrations of DS for the indicated periods of time, and cell viability was measured using a CCK-8 kit according to 
Table I. Primer sequences used for RT-qPCR.

\begin{tabular}{lll}
\hline Gene & \multicolumn{1}{c}{ Forward primer } & \multicolumn{1}{c}{ Reverse primer } \\
\hline SREBP1c & GGTTTTGAACGACATCGAAGA & CGGGAAGTCACTGTCTTGGT \\
aP2 & AGCCTTTCTCACCTGGAAGA & TTGTGGCAAAGCCCACTC \\
FAS & TGATGTGGAACACAGCAAGG & GGCTGTGGTGACTCTTAGTGATAA \\
GLUT4 & TGTGGCCTTCTTTGAGATTGG & CTGAAGAGCTCGGCCCACCAA \\
Adiponectin & GCAGAGATGGCACTCCTGGA & CCCTTCAGCTCCTGTCATTCC \\
Leptin & TCCAGAAAGTCCAGGATGACAC & CACATTTTGGGAAGGCAGG \\
HSL & GGAGCACTACAAACGCAACGA & TCGGCCACCGGTAAAGAG \\
$\beta$-actin & AGTAGACTGGTTGTATCGGG & AGCGTCATCAGGAGAAAGG \\
\hline
\end{tabular}

SREBP1c, sterol regulatory element binding protein-1c; aP2, activating protein 2; GLUT4, glucose transporter 4; HSL, hormone-sensitive lipase; LPL, lipoprotein lipase.

the manufacturer's instructions. Absorbance was measured at $450 \mathrm{~nm}$ on a microplate reader (Biochrom Anthos Zenyth 200; Biochrom Ltd., Cambridge, UK).

Oil Red $O$ staining. The differentiation of the cells was induced as described above. On day 6 , the cells were stained with Oil Red O, according to the manufacturer's instructions to visualize lipid accumulation in the cells. The intracellular lipid content was measured by extracting Oil Red $\mathrm{O}$ with isopropanol, and the absorbance at $520 \mathrm{~nm}$ was recorded using a spectrophotometer.

Triglyceride assay. Cellular triglyceride contents were measured using a commercial triglyceride assay kit (Triglyzyme test; Wako Pure Chemical Industries Ltd., Saitama, Japan), according to the instructions provided by the manufacturer. Briefly, the cells were washed twice with phosphate-buffered saline (PBS) and lysed in RIPA lysis buffer. Following centrifugation at 3,000 x $\mathrm{g}$ for $5 \mathrm{~min}$, the supernatants were assayed for the triglyceride and protein content. The triglyceride was content normalized to the protein concentration determined using bovine serum albumin as the standard.

Western blot analysis. The cells were lysed in ice-cold RIPA buffer for $20 \mathrm{~min}$ and centrifuged $(15,000 \mathrm{x} \mathrm{g})$ for $20 \mathrm{~min}$ at $4^{\circ} \mathrm{C}$. The protein concentration was measured using a bicinchoninic acid method. Lysates $(30 \mu \mathrm{g})$ were run on sodium dodecyl sulfate-polyacrylamide gel electrophoresis and transferred onto polyvinylidene difluoride (PVDF) membranes (Amersham Pharmacia Biotech Inc., Piscataway, NJ, USA). Subsequently, blocking was performed with 5\% skimmed milk in Tris-buffered saline containing 0.1\% Tween-20 (TBST) for $1 \mathrm{~h}$ at room temperature. The membranes were probed with primary antibodies as indicated at $4^{\circ} \mathrm{C}$ overnight, washed with TBST 4 times, and subsequently incubated with horseradish peroxidase-conjugated secondary antibody for $45 \mathrm{~min}$. The membranes were washed again 3 times with TBST and the proteins were visualized using an enhanced chemiluminescence detection kit (Amersham Pharmacia Biotech Inc.).
Isolation of total RNA and quantitative reverse transcription (RT-qPCR). Total RNA was extracted using TRIzol reagent, according to the manufacturer's recommendations. Isolated RNA $(1 \mu \mathrm{g} / \mu \mathrm{l})$ was used for cDNA synthesis using the SuperScript II kit. Aliquots of cDNA were amplified on the an ABI Real-Time PCR system from Applied Biosystems Inc. (Forster City, CA, USA) using the SYBR-Green Master Mix from Applied Biosystems. GAPDH was used as the invariant control. Primers specific for the genes examined are listed in Table I. The results were presented as levels of expression relative to that of the control.

Cell cycle analysis. The cells were harvested at the indicated time points following MDI stimulation with or without DS. Subsequently, the cells were fixed overnight with $70 \%$ ethanol at $-20^{\circ} \mathrm{C}$, washed twice with PBS, and stained with $50 \mu \mathrm{g} / \mathrm{ml}$ propidium iodide (IP) solution containing $25 \mu \mathrm{g} / \mathrm{ml}$ RNase A for $30 \mathrm{~min}$ at $37^{\circ} \mathrm{C}$. Cell cycle analysis was performed using the FACSCalibur flow cytometry system (BD Biosciences, San Diego, CA, USA), and data analysis was performed using FlowJo v10 software (TreeStar, Inc., Ashland, OR, USA).

Statistical analysis. All values are presented as the means \pm SEM. Statistical significance was determined using the Student's t-test. P-values $<0.05$ were considered to indicate statistically significant differences.

\section{Results}

Effect of DS on adipocyte viability. Several studies have demonstrated the cytotoxic effects of DS in a number of cell lines $(14,15)$. This prompted us to examine the possibility that the use of DS may result in cytotoxicity to 3T3-L1 cells. To examine this possibility, we examined the effects of DS on the viability of 3T3-L1 cells by CCK-8 assay. DS (0-4 $\mu \mathrm{M})$ showed no significant cytotoxicity towards the differentiating preadipocytes. However, $8 \mu \mathrm{M}$ DS inhibited the viability of the cells by approximately $50 \%$ at $24 \mathrm{~h}$ (Fig. $2 \mathrm{~A}$ and data not shown). Therefore, we selected the maximum dose of DS $(4 \mu \mathrm{M})$ for 
A
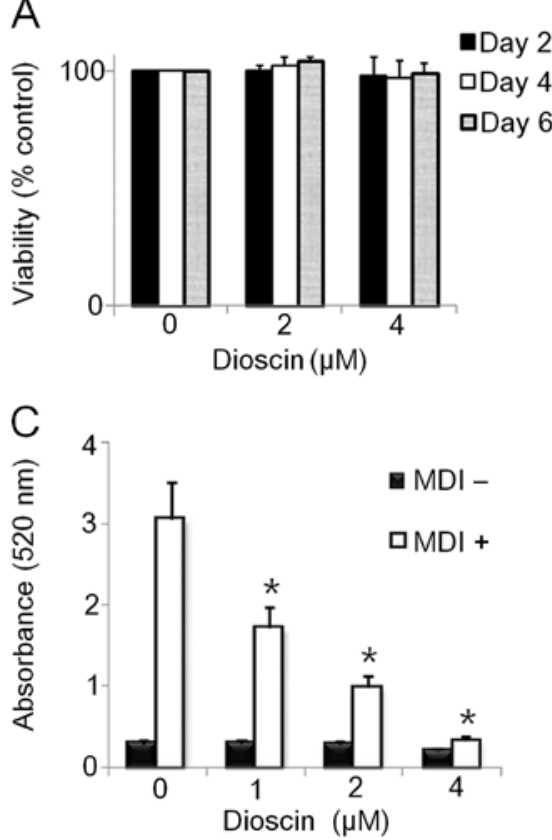

B
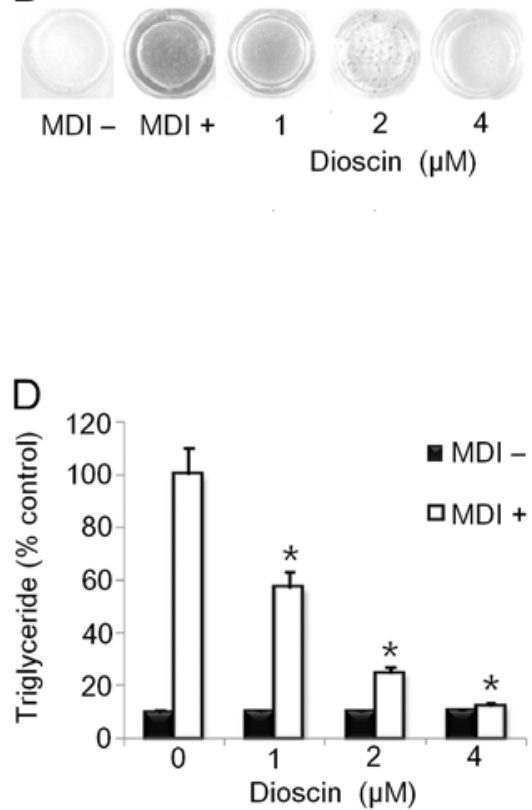

Figure 2. Dioscin (DS) inhibits adipogenesis in 3T3-L1 cells. 3T3-L1 preadipocytes were stimulated with insulin [multiple daily insulin (MDI)] to induce differentiation into adipocytes. (A) Post-confluent cells were incubated with MDI, with or without indicated concentrations of DS, for the indicated periods of time, and the viability of the cells was assessed by CCK-8 assay. (B) The cells were cultured in a differentiation medium with various concentrations of DS for 6 days and were subjected to Oil Red O (ORO) staining to analyze lipid accumulation. (C) The ORO-stained lipids were extracted using isopropanol, and the absorbance was measured using a spectrophotometer at $520 \mathrm{~nm}$. (D) After 6 days of differentiation, the cells were subjected to triglyceride content assay using a commercial kit. Data are presented as the means \pm SEM $\left({ }^{*} \mathrm{P}<0.05\right)$, and the experiments were performed at least 3 times with similar results.

A

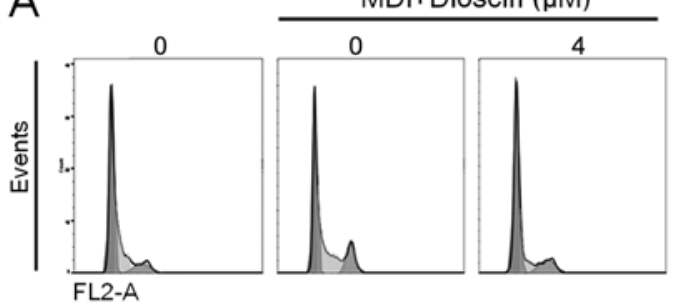

$\mathrm{B}$

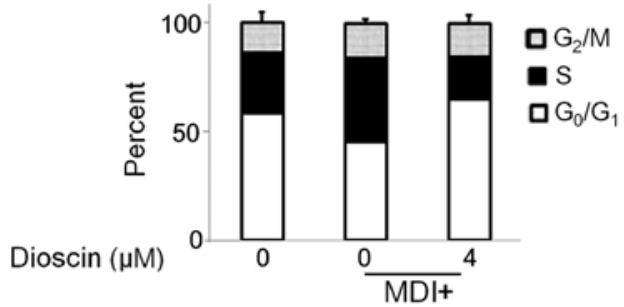

Figure 3. Cell cycle analysis of 3T3-L1 cells treated with dioscin (DS) during the mitotic clonal expansion (MCE) stage of adipogenesis. The differentiation of post-confluent preadipocytes was induced by insulin [multiple daily insulin (MDI)] with or without DS $(4 \mu \mathrm{M})$. (A) The cells were harvested after $48 \mathrm{~h}$ of stimulation with MDI and stained propidium iodide (PI) for flow cytometry. (B) The graphs represent the percentage of the cells in the $G_{0} / G_{1}, S$ or $\mathrm{G}_{2} \mathrm{M}$ phases of the cell cycle treated with or without DS. The experiment was performed 3 times with similar results.

further experiments on the effects of DS on adipogenesis to rule out the possibility that the inhibition of adipogenesis by DS may result from its cytotoxic effects on 3T3-L1 cells.

DS inhibits lipid accumulation in 3T3-L1 adipocytes. We examined the effects of DS on the differentiation of 3T3-L1 preadipocytes to adipocytes. The cells were stimulated with MDI to initiate differentiation. The culture medium was supplemented with various doses $(0-4 \mu \mathrm{M})$ of DS for 6 days. DS dose-dependently decreased intracellular fat accumulation compared to the control as demonstrated by morphological and quantitative analysis of intracellular lipids by Oil Red $\mathrm{O}$ staining (Fig. 2B and C). Consistent with these results, the triglyceride content in the 3T3-L1 cells treated with the indicated doses of DS was lower than that in the control cells differentiated for 6 days (Fig. 2D).

DS suppresses MCE during the early phase of adipogenesis. During adipogenesis, multiple rounds of cell cycle progression contribute to the MCE process. Therefore, we sought to examine the effects of DS on the cell cycle progression of 3T3-L1 cells during the MCE process. The results from flow cytometry revealed that the DS-treated cells showed a delayed cell cycle progression $48 \mathrm{~h}$ following stimulation with MDI (Fig. 3). The percentage of preadipocytes in the $\mathrm{G}_{0} / \mathrm{G}_{1}$ phase was approximately $58 \%$, while $45 \%$ of the untreated adipocytes and $65 \%$ of the DS-treated adipocytes were in the $\mathrm{G}_{0} / \mathrm{G}_{1}$ phase. These observations suggested that DS inhibits clonal expansion of the cells by inducing $G_{0} / G_{1}$ phase arrest.

DS inhibits the protein expression of adipogenic transcription factors. The differentiation of preadipocytes into adipocytes involves the sequential activation of several pro-adipogenic transcription factors, such as, $\mathrm{C} / \mathrm{EBP} \alpha / \beta / \delta$ and PPAR $\gamma$. Thus, we examined whether the reduced fat accumulation in the adipocytes was due to the downregulation of the aforementioned adipogenic transcription factors. As shown in Fig. 4, DS 


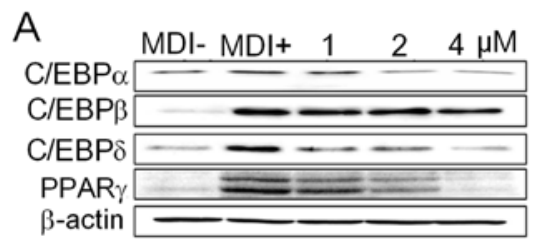

\section{$\mathrm{B}$}

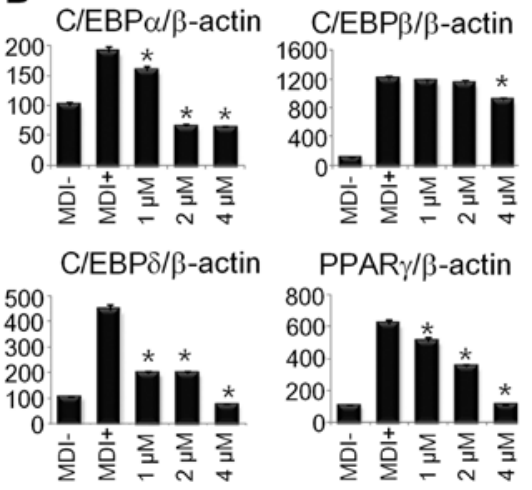

Figure 4. Dioscin (DS) inhibits the protein expression of differentiation-related transcription factors. (A) 3T3-L1 cells were cultured in the presence of insulin [multiple daily insulin (MDI)] with or without the indicated doses of DS for $48 \mathrm{~h}$, and protein extraction was performed for western blot analysis of the indicated transcription factors. (B) The bands were normalized to an internal control ( $\beta$-actin), and the graphs are presented as the relative ratio. Data represent the means \pm SEM $\left({ }^{*} \mathrm{P}<0.05\right)$ and the experiments were performed at least 3 times with similar results.
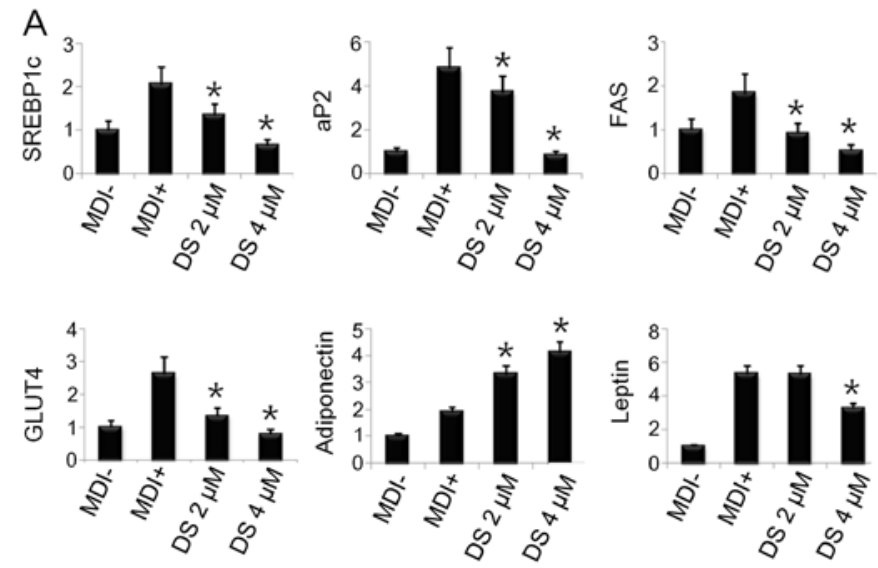

$\mathrm{B}$

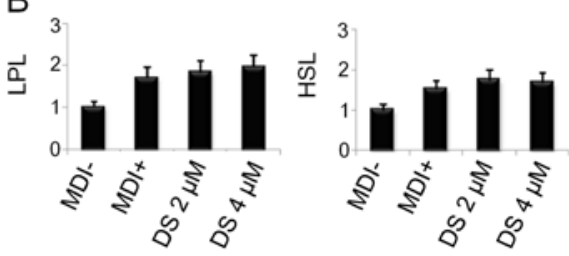

Figure 5. Effect of dioscin (DS) on the adipogenesis-related gene expression in 3T3-L1 cells. Preadipocytes were incubated with insulin [multiple daily insulin (MDI)] with or without the indicated doses of DS for $48 \mathrm{~h}$. (A) The expression of sterol regulatory element binding protein-1c (SREBP1c), activating protein 2 (aP2), fatty acid synthase (FAS), glucose transporter 4 (GULT4), adiponectin and leptin was assessed by RT-qPCR as described in the 'Materials and methods'. (B) The expression of the lipolytic genes, lipoprotein lipase (LPL) and hormone-sensitive lipase (HSL), was also analysed as described above. Data are presented as the means \pm SEM $\left({ }^{*} \mathrm{P}<0.05\right)$. The experiment was performed in triplicate.
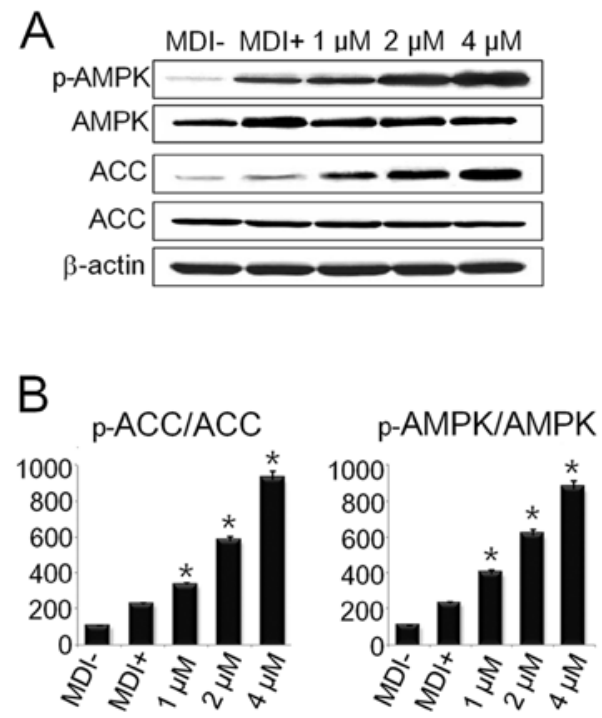

Figure 6. Dioscin (DS) enhances the phosphorylation of AMP-kinase (AMPK) and its target, acetyl-CoA carboxylase (ACC). 3T3-L1 cells were treated with the indicated concentrations of DS and stimulated with insulin [multiple daily insulin (MDI)] for $48 \mathrm{~h}$. (A) Subsequently, the protein expression of AMPK and ACC, phosphorylated and total, was analysed by western blot analysis, as described in the 'Materials and methods'. (B) The graphs show the band intensity ratio of the phosphorylated form by the total protein expression of AMPK and ACC. Data represent means \pm SEM $\left({ }^{*} \mathrm{P}<0.05\right)$, and the experiments were performed at least 3 times with similar results.

significantly inhibited the protein expression of $\mathrm{C} / \mathrm{EBP} \alpha / \beta / \delta$ and PPAR $\gamma$, suggesting that DS inhibits adipogenesis by suppressing the expression of adipogenic transcription factors.

DS inhibits adipogenesis-related gene expression in 3T3-L1 cells. As the adipogenic transcription factors were downregulated by DS, we further examined the expression of other adipogenesis-related genes involved in lipogenic and fatty acid oxidation and glucose homeostasis pathways. As shown in Fig. 5A, DS $(4 \mu \mathrm{M})$ significantly inhibited the mRNA expression of SREBP-1c, activating protein 2 (aP2), fatty acid synthase (FAS), glucose transporter 4 (GLUT4) and leptin. However, there was no significant difference in the mRNA levels of hormone-sensitive lipase (HSL), lipoprotein lipase (LPL) and adiponectin when compared to the controls (Fig. 5B).

DS regulates the AMPK pathway during adipogenesis. AMPK and its target, acetyl Co-A carboxylase (ACC), are the key regulators of preadipocyte differentiation and adipogenesis. Our results revealed that DS enhanced the phosphorylation of both AMPK and ACC (Fig. 6). These results suggest that DS regulates the adipogenic process in adipocytes through the AMPK pathway.

$D S$ regulates the MAPK pathway during adipogenesis. The suppression of ERK1/2 using pharmacological inhibitors has been shown to correlate with the inhibition of cell cycle progression, the partial blockage of MCE and the reduced expression of adipogenic factors (37). On the other hand, the pharmacological inhibition of the p38 pathway has been known to suppress adipogenesis in 3T3-L1 cells by downregulating C/EBP $\beta$ phosphorylation and its post-translational activation (20). Thus, we 


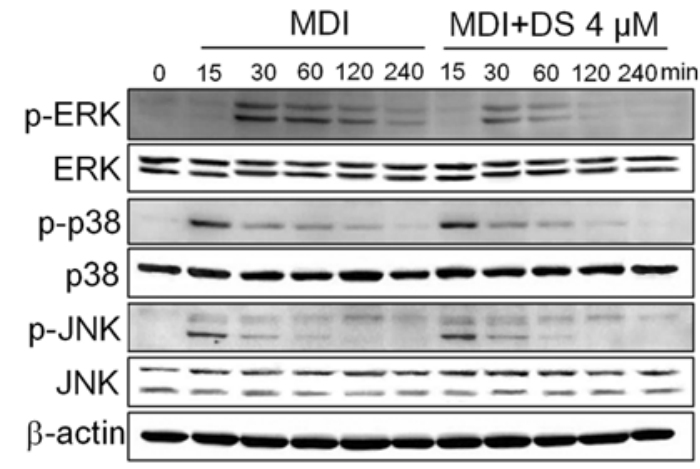

Figure 7. Effect of dioscin (DS) on the activation of the MAPK signaling pathway in 3T3-L1 adipocytes. The cells were treated with DS at the indicated concentrations during the insulin [multiple daily insulin (MDI)]-induced differentiation for $48 \mathrm{~h}$. ERK1/2, JNK and p38, and their phosphorylated forms were assessed by the western blot analysis. The experiments were performed at least 3 times and yielded similar results.
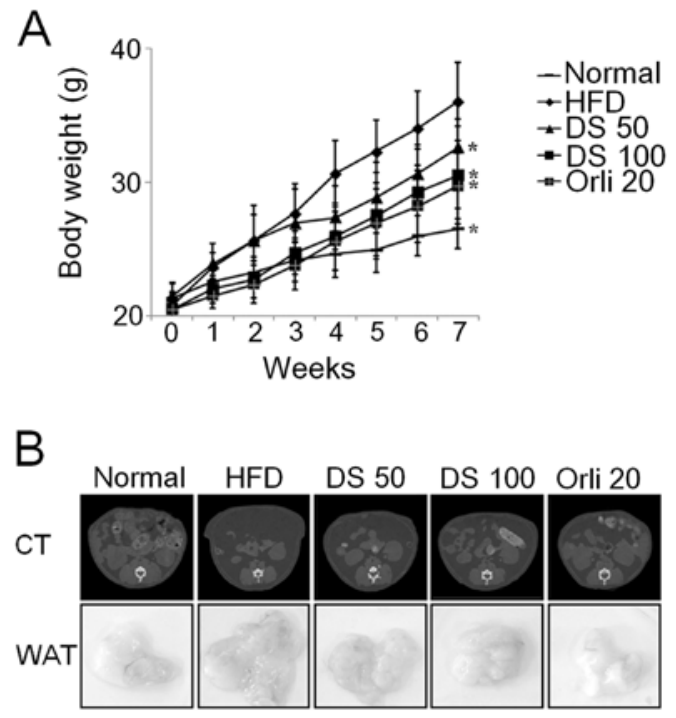

Figure 8. Effect of dioscin (DS) on body weight and fat accumulation in mice with high-fat diet (HFD)-induced obesity. Dose-dependent decrement of body weight in DS-treated mice fed HFD. (A) Graph depicts weight (in grams) of mice fed a normal diet (normal), HFD, HFD plus DS $50 \mathrm{mg} / \mathrm{kg}$ (DS 50), HFD plus DS $100 \mathrm{mg} / \mathrm{kg}$ (DS 100), or HFD plus orlistat (Orli) $20 \mathrm{mg} / \mathrm{kg}$ (Orli 20) for up to 7 weeks. (B) Representative micro-CT scanning images of abdominal cross-sections and macroscopic images of abdominal white adipose tissue (WAT) of the above-mentioned mouse groups. Data represent means \pm SEM $\left({ }^{*} \mathrm{P}<0.05\right), \mathrm{n}=8$ per group.

wished to determine whether DS inhibits ERK and p38 activation in 3T3-L1 cells. The results from western blot analysis revealed that DS inhibited the phosphorylation of the ERK and p38 pathways. However, we did not observe any inhibitory effect of DS on the phosphorylation of JNK (Fig. 7).

DS suppresses fat accumulation in mice with HFD-induced obesity. To assess the effects of DS on adipose tissue accumulation, we compared body weight and abdominal fat content in the different mouse groups. DS dose-dependently reduced body weight compared with the group fed the HFD and not treated with DS. There was a significant reduction in body weight after 4 weeks of DS $(50 \mathrm{mg} / \mathrm{kg})$ treatment in the mice HFD-induced obesity in comparison to the controls.
Moreover, treatment with DS (100 mg/kg) markedly inhibited body weight gain in comparison to the controls from the first weeks of treatment (Fig. 8A). This decrease in body weight was due to a significant decrease in fat accumulation as established by micro-CT scanning and macroscopy of abodominal fat (Fig. 8B).

\section{Discussion}

Obesity is a chronic, socially stigmatized and costly disease that has become an epidemic worldwide. The drugs currently available for the treatment of obesity have raised safety concerns and have demonstrated poor efficacy. For instance, orlistat is known to cause fetal fat loss and gastrointestinal symptoms. Thus, it is necessary to identify new medicinal products that are safe and effective in treating obesity $(21,22)$.

The increased growth and proliferation of adipocyte precursor cells lead to increased adipose mass, which represents the size and number of adipocytes. Our results revealed that DS at a dose of up to $4 \mu \mathrm{M}$ did not affect the viability of differentiating 3T3-L1 cells. This finding prompted us to eliminate the possibility that DS-induced cytotoxicity accounts for the inhibition of adipogenesis. Moreover, DS delayed the cell cycle progression in differentiating 3T3-L1 cells during the early phase of adipogenesis upon MDI stimulation. Further experiments demonstrated that DS dose-dependently reduced adipogenesis in 3T3-L1 cells, accompanied by a decreased triglyceride content.

PPARs are ligand-activated transcription factors that have 3 homologues: PPAR $\alpha / \beta / \gamma$, and have been reported to play a role in glucose and protein metabolism. They also regulate the proliferation and differentiation of preadipocytes (23-25). $\mathrm{C} / \mathrm{EBPs}$ are crucial proteins involved in preadipocyte differentiation. During the early steps of adipocyte differentiation steps, the activation of $\mathrm{C} / \mathrm{EBP} \beta$ and $\delta$ is known to result in the activation of $\mathrm{C} / \mathrm{EBP} \alpha$ and PPAR $\gamma$, which in turn control adipogenesis and insulin sensitivity in adipocytes (26-28). Moreover, the insulin-dependent glucose uptake requires the translocation of GLUT4, whose expression is regulated by C/EBPs from intracellular storage sites to the cell surface $(7,11,29)$. Another transcription factor, SREBP-1c, has been implicated in preadipocyte differentiation and fatty acid metabolism (6). $\mathrm{C} / \mathrm{EBP} \alpha$, PPAR $\gamma$ and SREBP-1c activation is known to regulate the adipocyte differentiation markers and genes associated with lipid metabolism, such as aP2, fatty acid synthase (FAS), LPL and HSL (30,31).

Our findings revealed that DS significantly downregulated $\mathrm{C} / \mathrm{EBP} \alpha / \beta / \delta$, PPAR $\gamma$, SREBP-1c and GLUT4 expression, which is essential for adipogenesis. Consistent with our results, previous studies have shown that ursolic acid and resveratrolamplified grape skin extracts inhibit adipogenesis in 3T3-L1 cells through the downregulation of PPAR $\gamma$ and C/EBP isoforms $(6,31)$. In accordance with our results, a previous study reported that 4-hydroxyderricin and xanthoangelol, plant-derived anti-obesity compounds, inhibited GLUT4 expression during the differentiation of 3T3-L1 cells (11). We also observed reduced mRNA levels of aP2 and FAS in adipocytes treated with DS, in accordance with a previous study showing the anti-adipogenic effects of resveratrol-amplified grape skin extracts on adipocytes (31). On the other hand, 
leptin is secreted by adipocytes, suppresses food intake and promotes energy expenditure. Thus, leptin levels are increased with adipogenesis and obesity $(32,33)$. In this study, decreased leptin levels in 3T3-L1 cells treated with DS indicated that DS exerted anti-adipogenic effects. Adiponectin, a vital hormone secreted by adipose tissue has been suggested to exert antidiabetic, anti-inflammatory and anti-atherogenic effects (32). In our study, the increased adiponectin expression in DS-treated 3T3-L1 cells was possibly the result of the anti-inflammatory effects of DS on adipocytes. A previous study suggested that high adiponectin production due to the presence of niacin is the result of its direct anti-inflammatory properties (34). However, further studies are required to demonstrate the direct anti-inflammatory properties of DS on adipocytes. LPL is an enzyme that hydrolyzes triglycerides in order to facilitate the fatty acid uptake by cells. On the other hand, HSL plays an important role in the mobilization of triacylglycerol stored inside cells (35). In this study, although there was a tendency towards an enhanced gene expression of LPL and HSL upon DS treatment in differentiating adipocytes, the difference was not statistically significant. However, this result may correlate with the reduced adipogenesis in DS-treated 3T3-L1 cells, possibly due to increased lipolysis in adipocytes.

Accumulating evidence suggests that AMPK is a major protein that regulates cellular energy homeostasis and regulates a number of biological pathways, such as carbohydrate and lipid metabolism. Hence, it is one of the most important targets for treating diabetes and obesity $(8-10,36)$. ACC is important for the synthesis and consumption of fatty acids and is a target of AMPK (11). In the present study, DS increased the phosphorylation of AMPK and ACC during preadipocyte differentiation. These results indicate that DS inhibits adipogenesis through the AMPK pathway. In accordance with our results, several compounds, such as chitin, ginsenosides, epigallocatchenin gallate and aspigenin have been reported to target AMPK for the inhibition of adipocyte differentiation (6).

Previous studies have indicated that the MAPKs, ERK1/2, JNK and p38, are involved in adipocyte differentiation (11). The downregulation of ERK1/2 or p38 by their inhibitors has been shown to result in reduced adipocyte differentiation $(20,37,38)$, suggesting that ERK1/2 and p38 are essential for adipogenesis. Consistent with this result, our data demonstrated that DS suppressed the phosphorylation of ERK1/2 and p38. However, we did not observed any effect on JNK activation upon DS treatment during adipocyte differentiation. There is limited evidence on the role of JNK in adipocyte differentiation (20). However, contrary to our findings, a previous study demonstrated that JNK phosphorylation is important for adipocyte differentiation and that JNK inhibitor (SP600125) reduced the lipid accumulation in adipocytes (11).

In the present study, we investigated the potential role of DS in regulating the adipogenesis of 3T3-L1 preadipocytes. We demonstrate that DS inhibits adipogenesis without exerting any cytotoxic effects on differentiating preadipocytes. This antiadipogenic effect targets the MCE phase, where DS retards cell cycle progression and decreases the expression of proadipogenic transcription factors. The DS-associated blockage of the MCE phase is accompanied by an inhibition of the phosphorylation of the MAPKS, ERK1/2 and p38. Moreover, DS induced the phosphorylation of AMPK. In our in vivo mouse model of obesity, DS significantly suppressed body weight gain and abdominal fat accumulation. This finding was consistent with previously reported results showing that treatment with Dioscorea plant extract reduced body weight and fat accumulation in mice with HFD-induced obesity $(18,19)$.

Taken together, our data demonstrate that DS is a natural anti-adipogenic molecule that targets the AMPK/MAPK pathway, inhibits the MCE phase and decreases the expression of adipogenic transcription factors during the process of adipogenesis in 3T3-L1 cells; it also modulates body weight and fat accumulation in mice with HFD-induced obesity.

\section{Acknowledgements}

The present study was financially supported by the Ministry of Knowledge Economy (MKE), Korea Institute for Advancement of Technology (KIAT) through the Inter-ER Cooperation Projects (R0002019).

\section{References}

1. Kopelman PG: Obesity as a medical problem. Nature 404: 635-643, 2000

2. Kwon JY, Seo SG, Heo YS, Yue S, Cheng JX, Lee KW and Kim KH: Piceatannol, natural polyphenolic stilbene, inhibits adipogenesis via modulation of mitotic clonal expansion and insulin receptor-dependent insulin signaling in early phase of differentiation. J Biol Chem 287: 11566-115678, 2012.

3. Green $\mathrm{H}$ and Kehinde O: An established preadipose cell line and its differentiation in culture II. Factors affecting the adipose conversion. Cell 5: 19-27, 1975.

4. Gregoire FM, Smas CM and Sul HS: Understanding adipocyte differentiation. Physiol Rev 78: 783-809, 1998.

5. Ntambi JM and Young-Cheul K: Adipocyte differentiation and gene expression. J Nutr 130: 3122S-3126S, 2000.

6. He Y, Li Y, Zhao T, Wang Y and Sun C: Ursolic acid inhibits adipogenesis in 3T3-L1 adipocytes through LKB1/AMPK pathway. PLoS One 8: e70135, 2013.

7. Lee JO, Lee SK, Kim JH, et al: Metformin regulates glucose transporter 4 (GLUT4) translocation through AMP-activated protein kinase (AMPK)-mediated $\mathrm{Cbl} / \mathrm{CAP}$ signaling in 3T3-L1 preadipocyte cells. J Biol Chem 287: 44121-44129, 2012.

8. Jones RG, Plas DR, Kubek S, et al: AMP-activated protein kinase induces a p53-dependent metabolic checkpoint. Mol Cell 18: 283-293, 2005.

9. Luo Z, Saha AK, Xiang X and Ruderman NB: AMPK, the metabolic syndrome and cancer. Trends Pharmacol Sci 26: 69-76, 2005.

10. Song XM, Fiedler M, Galuska D, et al: 5-Aminoimidazole 4-carboxamide ribonucleoside treatment improves glucose homeostasis in insulin-resistant diabetic (ob/ob) mice. Diabetologia 45: $56-65,2002$.

11. Zhang T, Sawada K, Yamamoto $\mathrm{N}$ and Ashida $\mathrm{H}$ : 4-Hydroxyderricin and xanthoangelol from Ashitaba (Angelica keiskei) suppresses differentiation of preadipocytes to adipocytes via AMPK and MAPK pathways. Mol Nutr Food Res 57: 1729-1740, 2013

12. Xiao J, Wang NL, Sun B and Cai GP: Estrogen receptor mediates the effects of pseudoprotodiocsin on adipogenesis in 3T3-L1 cells. Am J Physiol Cell Physiol 299: C128-C138, 2010.

13. Nakamura T, Komori C, Lee Y, Hashimoto F, Yahara S, Nohara T and Ejima A: Cytotoxic activities of solanum steroidal glycosides. Biol Pharm Bull 19: 564-566, 1996.

14. Cai J, Liu M, Wang Z and Ju Y: Apoptosis induced by dioscin in Hela cells. Biol Pharm Bull 25: 193-196, 2002.

15. Liu MJ, Wang Z, Ju Y, Zhou JB, Wang Y and Wong RN: The mitotic-arresting and apoptosis-inducing effects of diosgenyl saponins on human leukemia cell lines. Biol Pharm Bull 27: 1059-1065, 2004

16. Sata N, Matsunaga S, Fusetani N, Nishikawa H, Takamura S and Saito T: New antifungal and cytotoxic steroidal saponins from the bulbs of an elephant garlic mutant. Biosci Biotechnol Biochem 62: 1904-1911, 1998. 
17. Ikeda T, Ando J, Miyazono A, et al: Anti-herpes virus activity of Solanum steroidal glycosides. Biol Pharm Bull 23: 363-364, 2000.

18. Kwon CS, Sohn HY, Kim SH, et al: Anti-obesity effect of Dioscorea nipponica Makino with lipase-inhibitory activity in rodents. Biosci Biotechnol Biochem 67: 1451-1456, 2003.

19. Song MY, Lv N, Kim EK, et al: Antiobesity activity of aqueous extracts of Rhizoma Dioscoreae Tokoronis on high-fat diet-induced obesity in mice. J Med Food 12: 304-309, 2009.

20. Bost F, Aouadi M, Caron L and Binétruy B: The role of MAPKs in adipocyte differentiation and obesity. Biochimie 87: 51-56, 2005.

21. Bray GA and Tartaglia LA: Medicinal strategies in the treatment of obesity. Nature 404: 672-677, 2000.

22. Bray GA: A concise review on the therapeutics of obesity. Nutrition 16: 953-960, 2000.

23. Christodoulides C and Vidal-Puig A: PPARs and adipocyte function. Mol Cell Endocrinol 318: 61-68, 2010.

24. Takahashi S, Tanaka T, Kodama T and Sakai J: Peroxisome proliferator-activated receptor $\delta$ (PPAR $\delta$ ), a novel target site for drug discovery in metabolic syndrome. Pharmacol Res 53: 501-507, 2006.

25. Tontonoz P, Hu E and Spiegelman BM: Stimulation of adipogenesis in fibroblasts by PPAR gamma 2, a lipid-activated transcription factor. Cell 79: 1147-1156, 1994

26. Darlington GJ, Ross SE and MacDougald OA: The role of $\mathrm{C} / \mathrm{EBP}$ genes in adipocyte differentiation. J Biol Chem 273: 30057-30060, 1998.

27. Cao Z, Umek RM and McKnight SL: Regulated expression of three C/EBP isoforms during adipose conversion of 3T3-L1 cells. Genes Dev 5: 1538-1552, 1991.

28. Wu Z, Rosen ED, Brun R, et al: Cross-regulation of C/EBP alpha and PPAR gamma controls the transcriptional pathway of adipogenesis and insulin sensitivity. Mol Cell 3: 151-158, 1999.
29. Lu C, Zhu W, Shen CL and Gao W: Green tea polyphenols reduce body weight in rats by modulating obesity-related genes. PLoS One 7: e38332, 2012

30. Warnke I, Goralczyk R, Fuhrer E and Schwager J: Dietary constituents reduce lipid accumulation in murine $\mathrm{C} 3 \mathrm{H} 10 \mathrm{~T} 1 / 2$ adipocytes: a novel fluorescent method to quantify fat droplets. Nutr Metab (Lond) 8: 30, 2011.

31. Zhang XH, Huang B, Choi SK and Seo JS: Anti-obesity effect of resveratrol-amplified grape skin extracts on 3T3-L1 adipocytes differentiation. Nutr Res Pract 6: 286-293, 2012.

32. Yuan G, Jia J, Di L, et al: Effects of C-reactive protein on adipokines genes expression in 3T3-L1 adipocytes. Biochem Biophys Res Commun 424: 462-468, 2012.

33. Alvala R, Alvala M, Sama V, Dharmarajan S, Ullas JV and B MR: Scientific evidence for traditional claim of anti-obesity activity of Tecomella undulata bark. J Ethnopharmacol 148: 441-448, 2013.

34. Wanders D, Graff EC, White BD and Judd RL: Niacin increases adiponectin and decreases adipose tissue inflammation in high fat diet-fed mice. PLoS One 8: e71285, 2013.

35. Kanagasabapathy G, Malek SN, Mahmood AA, Chua KH, Vikineswary S and Kuppusamy UR: Beta-glucan-rich extract from Pleurotus sajor-caju (Fr.) Singer prevents obesity and oxidative stress in C57BL/6J mice fed on a high-fat diet. Evid Based Complement Alternat Med 2013: 185259, 2013.

36. Zhang BB, Zhou G and Li C: AMPK: an emerging drug target for diabetes and the metabolic syndrome. Cell Metab 9: 407-416, 2009.

37. Tang QQ, Otto TC and Lane MD: Mitotic clonal expansion: a synchronous process required for adipogenesis. Proc Natl Acad Sci USA 100: 44-49, 2003.

38. Engelman JA, Lisanti MP and Scherer PE: Specific inhibitors of p38 mitogen-activated protein kinase block 3T3-L1 adipogenesis. J Biol Chem 273: 32111-32120, 1998. 